\title{
Prorenoate Potassium
}

National Cancer Institute

\section{Source}

National Cancer Institute. Prorenoate Potassium. NCI Thesaurus. Code C84118.

The potassium salt form of prorenoate, a steroidal aldosterone antagonist with potassium-sparing diuretic activity. Prorenoate antagonizes the activity of aldosterone at the mineralocorticoid receptor in the nephron and thereby prevents sodium reabsorption. This interferes with the sodium/potassium exchanger and causes sodium and water excretion while reducing potassium excretion. 